\title{
Investigation of effects of assisted ion bombardment on mechanical loss of sputtered tantala thin films for gravitational wave interferometers
}

\author{
Le Yang $\odot,{ }^{1, *}$ Emmett Randel, ${ }^{2}$ Gabriele Vajente, ${ }^{3}$ Alena Ananyeva, ${ }^{3}$ Eric Gustafson, ${ }^{3}$ Ashot Markosyan, ${ }^{4}$ \\ Riccardo Bassiri, ${ }^{4}$ Martin M. Fejer, ${ }^{4}$ and Carmen S. Menoni ${ }^{1,2,}$ \\ ${ }^{1}$ Department of Chemistry, Colorado State University, Fort Collins, Colorado 80523, USA \\ ${ }^{2}$ Department of Physics, Colorado State University, Fort Collins, Colorado 80523, USA \\ ${ }^{3}$ LIGO Laboratory, California Institute of Technology, Pasadena, California 91125, USA \\ ${ }^{4}$ Edward L. Ginzton Laboratory, Stanford University, Stanford, California 94305, USA
}

(Received 11 June 2019; published 11 December 2019)

\begin{abstract}
Reduction of Brownian thermal noise due to mechanical loss in high-reflectivity mirror coatings is critical for improving the sensitivity of future gravitational wave detectors. In these mirrors, the mechanical loss at room temperature is dominated by the high refractive index component, amorphous tantala $\left(\mathrm{Ta}_{2} \mathrm{O}_{5}\right)$ or tantala doped with titania $\left(\mathrm{Ti}: \mathrm{Ta}_{2} \mathrm{O}_{5}\right)$. Toward the goal of identifying mechanisms that could alter mechanical loss, this work investigates the use of assist ion bombardment in the reactive ion beam sputtering deposition of tantala single layers. Low-energy assist ion bombardment can enhance adatom diffusion. Low-energy assist $\mathrm{Ar}^{+}$and $\mathrm{Xe}^{+}$ion bombardment at different conditions was implemented during deposition to identify trends in the mechanical loss with ion mass, ion energy, and ion dose. It is shown that the atomic structure and bonding states of the tantala thin films are not significantly modified by low-energy assist ion bombardment. The coatings mechanical loss remains unaltered by ion bombardment within errors. Based on an analysis of surface diffusivity, it is shown that the dominant deposition of tantala clusters and limited surface diffusion length of oxygen atoms constrain structural changes in the tantala films. A slower deposition rate coupled with a significant increase in the dose of the low-energy assist ions may provide more favorable conditions to improve adatom diffusivity.
\end{abstract}

DOI: 10.1103/PhysRevD.100.122004

\section{INTRODUCTION}

Gravitational waves emerge from energetic processes of astrophysical bodies in the Universe. To detect a gravitational wave strain $(\Delta L / L)$ of $10^{-23}$ or smaller, gravitational wave detectors based on kilometer-scale interferometers have been established [1-3]. In the midband (approximately $100 \mathrm{~Hz}$ ), in which the Advanced LIGO (aLIGO) detector is most sensitive, Brownian thermal noise from the coatings in the test masses is the dominant source of noise $[4,5]$.

In aLIGO, the coatings on the test masses consist of alternating layers of amorphous silica $\left(\mathrm{SiO}_{2}\right)$ and titaniadoped tantala $\left(\mathrm{Ti}: \mathrm{Ta}_{2} \mathrm{O}_{5}\right)$ deposited by ion beam sputtering with less than $1 \mathrm{ppm}$ optical absorption loss at $1064 \mathrm{~nm}$ [6-8]. The thermal noise of the coatings is estimated from the internal mechanical losses of the layer materials based on the fluctuation-dissipation theorem $[9,10]$. It is well established that the coatings loss angle is dominated by the high-index $\mathrm{Ti}: \mathrm{Ta}_{2} \mathrm{O}_{5}$ layer $\left(2.9 \times 10^{-4} \mathrm{rad}\right)$ rather than the low-index $\mathrm{SiO}_{2}$ layer $\left(1.1 \times 10^{-5} \mathrm{rad}\right)$ [11]. A successful

\footnotetext{
*yangle@colostate.edu

†carmen.menoni@colostate.edu
}

strategy that has been employed to reduce the loss angle in tantala is to incorporate impurities, such as $\mathrm{Ti}, \mathrm{Zr}, \mathrm{Nb}$, and $\mathrm{Hf}$ [12]. Modeling of the atomic structure of Ti doped and undoped $\mathrm{Ta}_{2} \mathrm{O}_{5}$ showed that the doping modifies the medium-range order that correlates with reduced mechanical loss [13].

In this work, we explore the use of low-energy assist ion bombardment during the reactive sputtered deposition of ion beam sputtered (IBS) tantala thin films to investigate whether it would be possible to alter surface diffusion of particles (representing atoms, molecules, and clusters) sputtered from the target and chemi-sorbed on the growing tantala films. Enhanced surface mobility has been claimed to favor an atomic network that is thermodynamically more stable. Glasses with unprecedented thermodynamic stability enabled by high adatom mobility are reported in Ref. [14]. Work on amorphous $\mathrm{Si}$ (a-Si) deposited at elevated temperature also indicates suppressed mechanical loss at low temperature [15] and at room temperature [16], presumably via increased surface mobility. With the specific goal of enhancing surface mobility as observed in a-Si, tantala thin films were deposited by Vajente et al. with IBS at elevated temperatures [17]. The loss angle of the asdeposited tantala coatings reduced from around $16 \times 10^{-4}$ 
to $6 \times 10^{-4} \mathrm{rad}$ when the substrate temperature increased from $30^{\circ} \mathrm{C}$ to $400{ }^{\circ} \mathrm{C}$. Nevertheless, a major reduction in loss angle to $4 \times 10^{-4}$ rad was achieved with postannealing at $500^{\circ} \mathrm{C}$ independent of the initial deposition temperature. Alternatively, assist ion bombardment can lead to increased surface mobility. Previous work has shown that low-energy (i.e., $65 \mathrm{eV}$ ) bombardment using $\mathrm{He}^{+}, \mathrm{Ar}^{+}$, and $\mathrm{Xe}^{+}$ions increased the surface diffusion of $\mathrm{Ge}$ on $\operatorname{Si}(111)[18,19]$. Atomic rearrangement in $\mathrm{Ge}$ was also achieved during the thermal spike induced by $\mathrm{Ar}^{+}$ion bombardment [20].

In the results described herein, amorphous tantala thin films were deposited by reactive IBS with concurrent lowenergy assist $\mathrm{Ar}^{+}$and $\mathrm{Xe}^{+}$ion bombardment, respectively. The assist ion beam was operated at conditions that altered the assist ion energy and dose at the surface of the growing tantala film. It is shown that the atomic structure and bonding states of the tantala thin films are not significantly modified by the assist ion bombardment. Assessment of the room temperature mechanical loss shows that loss remains unaltered by low-energy ion bombardment within experimental errors. An analysis of mass transfer surface diffusivity shows that at the assist conditions of the experiment reordering by ion-assisted diffusion is negligible due to low coverage of mobile adatoms and the high diffusion activation energy of clusters.

\section{METHODS}

\section{A. Thin film deposition}

Tantala thin films were prepared using a Veeco Spector ${ }^{\circledR}$ dual IBS system. The system was baked at $150^{\circ} \mathrm{C}$ for $1 \mathrm{~h}$ and pumped down to $2.6 \times 10^{-5} \mathrm{~Pa}$ before deposition to reduce water partial pressure to below $10^{-6} \mathrm{~Pa}$. The main ion beam source using $\mathrm{Ar}^{+}$sputtered a Ta target in a reactive oxygen atmosphere at different beam voltages and beam currents that altered the deposition rate. In the absence of assist bombardment and when keeping the chamber temperature at $60^{\circ} \mathrm{C}$, the substrate reached an equilibrium temperature of approximately $100{ }^{\circ} \mathrm{C}$. The assist ion beam source was operated using either $\mathrm{Ar}^{+}$or $\mathrm{Xe}^{+}$to concurrently bombard the growing film at normal incidence. The substrate was positioned on a fixed rotation stage that at all times faces the assist ion source. The rotation speed was set at $4 \pi \mathrm{rad} / \mathrm{s}$. The temperature of the film/substrate in this case increases to approximately $130^{\circ} \mathrm{C}$ when using $100 \mathrm{eV}$ bombarding ions. Two $75 \mathrm{~mm}$ diameter, $1 \mathrm{~mm}$ thick fused silica disks were coated for mechanical loss measurements. Using the same conditions, the tantala films were deposited on $25.4 \mathrm{~mm}$ diameter fused-silica and silicon (100) substrates for optical and structural characterization.

Two control tantala thin film samples were deposited using the main ion source at different conditions with no assist ion beam (Table I). The deposition rate decreased from 3.6 (c1) to $0.9 \AA / \mathrm{s}$ (c2) when the main ion beam voltage and beam current were lowered. These deposition rates are indicative of complete oxide coverage of the Ta target [21]. Nevertheless, the sputtered particles that reach the substrate are a combination of Ta atoms and tantalum oxide fragments, the majority of which were found to be $\mathrm{Ta}_{2} \mathrm{O}_{2}$ ring clusters [22]. The variation in the deposition rate was used to control the arrival ratio between particles sputtered from the Ta target and the assist ions dose at the substrate surface.

Two sets of runs were carried out with either $\mathrm{Ar}^{+}$or $\mathrm{Xe}^{+}$ assist bombardment. In each set, the assist ion energy and dose were modified by varying the assist ion beam voltage and current. Details of the deposition conditions are in Tables II and III. The number of particles arriving at the substrate was estimated from the deposition rate of the control samples assuming sputtering of $\mathrm{Ta}_{2} \mathrm{O}_{2}$ clusters. For $\mathrm{c} 1$, with a deposition rate of $3.6 \AA / \mathrm{s}$, the number of particles arriving at the substrate was approximately $2.8 \times 10^{15}$ particles $/\left(\mathrm{cm}^{2} \cdot \mathrm{s}\right)$. The assist ion dose at the sample surface was measured with a Faraday cup. For an assist ion beam at $100 \mathrm{~V}$ and $100 \mathrm{~mA}$, the ion dose was

TABLE I. Deposition conditions and characterization results of control samples.

\begin{tabular}{|c|c|c|c|c|c|c|c|}
\hline Sample & $\begin{array}{l}\text { Main beam } \\
\text { voltage }(\mathrm{V})\end{array}$ & $\begin{array}{l}\text { Main beam } \\
\text { current }(\mathrm{mA})\end{array}$ & $\begin{array}{l}\text { Assist beam } \\
\text { voltage }(\mathrm{V})\end{array}$ & $\begin{array}{l}\text { Assist beam } \\
\text { current }(\mathrm{mA})\end{array}$ & $\begin{array}{c}\text { Deposition } \\
\text { rate }(\AA / s)\end{array}$ & $\begin{array}{l}\text { Thickness } \\
(\mathrm{nm})\end{array}$ & $\begin{array}{c}\text { Absorption loss } \\
(\mathrm{ppm})\end{array}$ \\
\hline $\mathrm{c} 1$ & 1250 & 600 & Not applicable & Not applicable & 3.6 & $523.6 \pm 1.9$ & $22.1 \pm 0.7$ \\
\hline $\mathrm{c} 2$ & 1000 & 300 & Not applicable & Not applicable & 0.9 & $503.8 \pm 1.2$ & $9.3 \pm 0.2$ \\
\hline
\end{tabular}

TABLE II. Deposition conditions and characterization results of samples prepared with $\mathrm{Ar}^{+}$assist.

\begin{tabular}{lccccccrr}
\hline \hline Sample & $\begin{array}{c}\text { Main beam } \\
\text { voltage }(\mathrm{V})\end{array}$ & $\begin{array}{c}\text { Main beam } \\
\text { current }(\mathrm{mA})\end{array}$ & $\begin{array}{c}\text { Assist beam } \\
\text { voltage }(\mathrm{V})\end{array}$ & $\begin{array}{c}\text { Assist beam } \\
\text { current }(\mathrm{mA})\end{array}$ & $\begin{array}{c}\text { Deposition } \\
\text { rate }(\AA / \mathrm{s})\end{array}$ & $\begin{array}{c}\text { Thickness } \\
(\mathrm{nm})\end{array}$ & $\begin{array}{c}\text { Absorption } \\
\text { loss (ppm) }\end{array}$ & $\begin{array}{c}\text { Particle-to-ion } \\
\text { arrival ratio }\end{array}$ \\
\hline s11 & 1250 & 600 & 100 & 100 & 3.5 & $479.2 \pm 1.7$ & $7.2 \pm 0.4$ & $22 / 1$ \\
s12 & 1250 & 600 & 100 & 200 & 3.4 & $487.0 \pm 1.9$ & $10.3 \pm 0.4$ & $10 / 1$ \\
s13 & 1000 & 300 & 100 & 100 & 0.8 & $505.6 \pm 2.2$ & $13.1 \pm 0.2$ & $5 / 1$ \\
\hline \hline
\end{tabular}


TABLE III. Deposition conditions and characterization results of samples prepared with $\mathrm{Xe}^{+}$assist.

\begin{tabular}{lcccccccc}
\hline \hline Sample & $\begin{array}{c}\text { Main beam } \\
\text { voltage (V) }\end{array}$ & $\begin{array}{c}\text { Main beam } \\
\text { current (mA) }\end{array}$ & $\begin{array}{c}\text { Assist beam } \\
\text { voltage (V) }\end{array}$ & $\begin{array}{c}\text { Assist beam } \\
\text { current (mA) }\end{array}$ & $\begin{array}{c}\text { Deposition } \\
\text { rate (A/s) }\end{array}$ & $\begin{array}{c}\text { Thickness } \\
(\mathrm{nm})\end{array}$ & $\begin{array}{c}\text { Absorption } \\
\text { loss (ppm) }\end{array}$ & $\begin{array}{c}\text { Particle-to-ion } \\
\text { arrival ratio }\end{array}$ \\
\hline s21 & 1250 & 600 & 50 & 100 & 3.3 & $496.0 \pm 2.1$ & $9.4 \pm 0.2$ & $40 / 1$ \\
s22 & 1250 & 600 & 50 & 200 & 3.3 & $496.0 \pm 2.2$ & $5.5 \pm 0.1$ & $23 / 1$ \\
s23 & 1000 & 300 & 50 & 200 & 0.8 & $469.8 \pm 1.6$ & $12.1 \pm 0.4$ & $5 / 1$ \\
s24 & 1000 & 300 & 100 & 100 & 0.8 & $490.4 \pm 1.8$ & $32.8 \pm 1.3$ & $4 / 1$ \\
\hline \hline
\end{tabular}

approximately $1.2 \times 10^{14} \mathrm{ions} /\left(\mathrm{cm}^{2} \cdot \mathrm{s}\right)$ for $\mathrm{Ar}^{+}$and approximately $1.6 \times 10^{14} \mathrm{ions} /\left(\mathrm{cm}^{2} \cdot \mathrm{s}\right)$ for $\mathrm{Xe}^{+}$. The particle-to-ion arrival ratio calculated from these quantities is shown in Tables II and III.

\section{B. Optical characterization}

The thickness and optical constants of the tantala films were obtained with a Horiba UVISEL spectroscopic ellipsometer. Thin films were scanned with a $60^{\circ}$ incident angle from 190 to $2101 \mathrm{~nm}$. The films thickness determined from the fitting had a standard deviation of approximately $2 \mathrm{~nm}$ for all samples. The coatings optical absorption loss at $1064 \mathrm{~nm}$ was measured by the photothermal common-path interferometry technique, which has sensitivity better than 1 ppm [23].

\section{X-ray diffraction and $x$-ray photoelectron spectroscopy}

Grazing angle x-ray diffraction (GAXRD) was used to characterize the atomic structure of the tantala coatings. A Bruker D8 Discover diffractometer with a grazing angle setup was used. An incident angle of approximately $1.5^{\circ}$ was used to minimize the diffraction signal from the substrate. The diffracted beam was collected by a scintillation detector as a function of 2theta $(2 \theta)$.

The bonding states of Ta in the samples coated on p-type (100) Si wafers were examined with a PE-5800 x-ray photoelectron spectrometer (XPS). The takeoff angle of $45^{\circ}$ was used for all scans. The neutralizer operating at $10 \mu \mathrm{A}$ was used to counteract the charging effect of the sample due to loss of electrons. The $\mathrm{C} 1 \mathrm{~s}$ peak position was used to calibrate the binding energy scale of the spectra. Highresolution spectra were collected for Ta $4 f, \mathrm{O} 1 s$, and $\mathrm{C} 1 s$. A superposition curve fitting procedure was used to analyze the spectra. Peak position and widths were determined from a least-square fitting using MultiPak. The peak shapes were fitted with Gaussian functions. The oxidation state of Ta is represented by the Ta $4 f 5 / 2$ and Ta $4 f 7 / 2$ doublet peak position. When fitting the acquired data, the energy separation of the doublet lines was fixed to $1.9 \mathrm{eV}$, and the area ratio was fixed to $1.3-1.33$.

\section{Coating mechanical loss}

Each coatings loss angle was assessed with the coating ringdown system developed at California Institute of
Technology [24]. The tantala films for this purpose were deposited on fused silica disks $75 \mathrm{~mm}$ in diameter and $1 \mathrm{~mm}$ in thickness. The system is based on the gentle nodal suspension method. After excitation of the disk modes, the amplitude of the motion at the mode peak frequency was tracked over time. The damping time is directly linked to the total mechanical loss, and the loss angle for the coating can be extracted. Uncoated substrates have a loss angle of $10^{-7}$ to $10^{-8} \mathrm{rad}$, which is orders of magnitude smaller than that of the tantala coatings. The coatings loss angle was measured both in as-deposited and annealed tantala thin films. The annealing procedure includes 1) a $5 \mathrm{~h}$ ramp up to $500{ }^{\circ} \mathrm{C}, 2$ ) a $10 \mathrm{~h}$ soaking at $500^{\circ} \mathrm{C}$, and 3) a $5 \mathrm{~h} \mathrm{ramp} \mathrm{down}$ to room temperature.

The results are presented in the following section.

\section{RESULTS AND DISCUSSION}

\section{A. Optical properties}

All tantala coatings are optically transparent. Non-ionassisted controlled films $\mathrm{c} 1$ and $\mathrm{c} 2$ have refractive indices of 2.10 and 2.09 at $1064 \mathrm{~nm}$, respectively. Similar values ranging from 2.09 to 2.11 were obtained for the tantala samples bombarded with $\mathrm{Ar}^{+}$or $\mathrm{Xe}^{+}$assist.

Low optical absorption loss at $1064 \mathrm{~nm}$ is a requirement for the tantala thin films to ensure that when used in the high reflectors they meet the stringent absorption loss (approximately $0.5 \mathrm{ppm}$ ) required for aLIGO coatings [25]. For most of the approximately $500 \mathrm{~nm}$ thick samples, an absorption loss of less than $18 \mathrm{ppm}$ at $1064 \mathrm{~nm}$ was measured (Tables I, II, and III). Preferential sputtering of oxygen by $\mathrm{Ar}^{+}$or $\mathrm{Xe}^{+}$was not evident at the low ion beam voltages used. One exception is s24, which has an absorption loss of $32.8 \mathrm{ppm}$. The minimum absorption, $5.5 \mathrm{ppm}$, was found in sample s 21 prepared with a $50 \mathrm{eV}$ $\mathrm{Xe}^{+}$assist beam.

\section{B. Structural and bonding properties}

GAXRD spectra of tantala coatings show an amorphous packing (Fig. 1). The arrows in Fig. 1 indicate the theoretical peak positions for the crystalline orthorhombic phase of $\mathrm{Ta}_{2} \mathrm{O}_{5}$ (Powder Diffraction File (PDF) 01-0829637) [26]. There are two main broad peaks between $20^{\circ}$ and $40^{\circ}$ and between $40^{\circ}$ and $70^{\circ}$. Signals from the fused silica substrate contribute to the profile at $2 \theta=20.8^{\circ}$. Between the control sample c2, sample s23 bombarded 


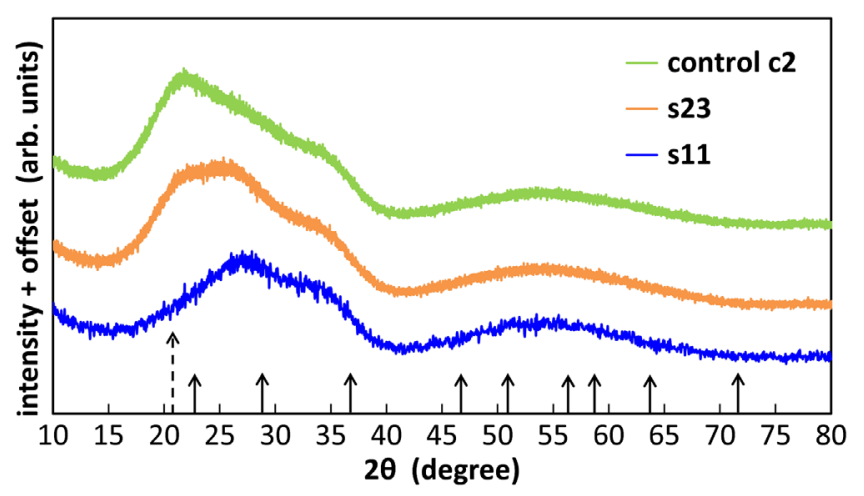

FIG. 1. Grazing angle x-ray diffraction spectra for control sample c2, sample s23 bombarded with $\mathrm{Xe}^{+}$assist, and sample s11 bombarded with $\mathrm{Ar}^{+}$. Signals from the fused silica substrate contribute to the peak at $20.8^{\circ}$, as indicated by the dashed arrow. Solid arrows indicate peaks from crystalline orthorhombic $\mathrm{Ta}_{2} \mathrm{O}_{5}$.

with assist $\mathrm{Xe}^{+}$, and sample s11 bombarded with $\mathrm{Ar}^{+}$, there are no significant differences in the spectra. Figure 1 shows that the atomic structure remained amorphous under concurrent ion bombardment, regardless of variance in the ion type, ion energy, or ion dose.

The XPS spectra of tantala provide information on the bonding states of Ta in as-deposited samples. As shown in Fig. 2, close agreement between measured spectra and peak synthesis was obtained. To evaluate the oxidation states, we used a $267 \mathrm{~nm}$ thick reference sample deposited using the
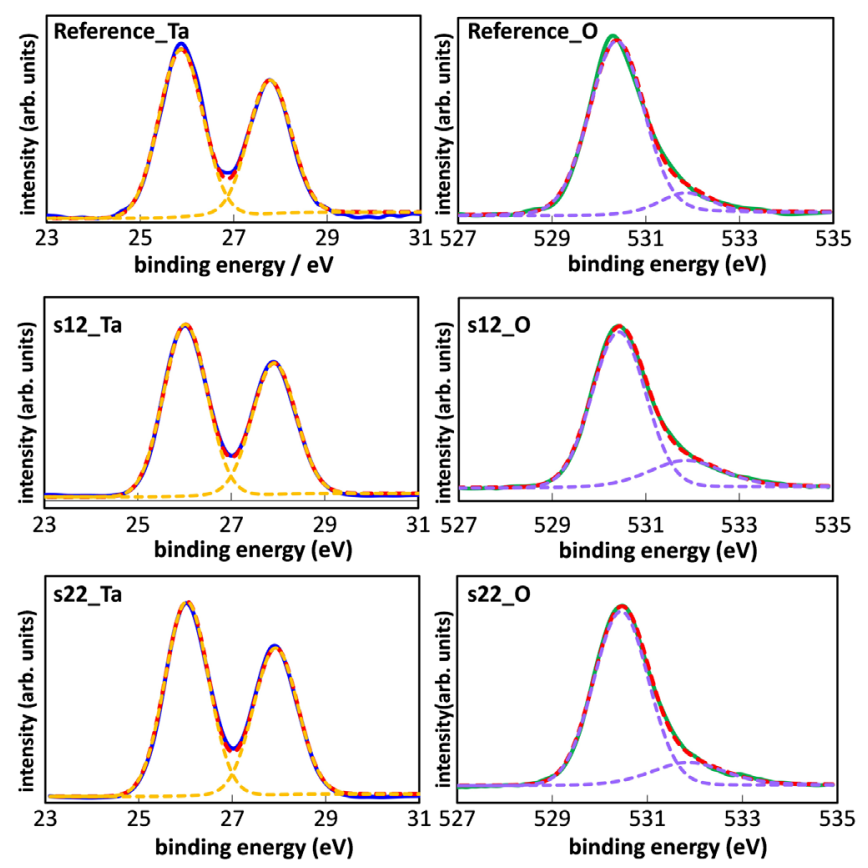

FIG. 2. Photoelectron spectra of Ta $4 f$ and $\mathrm{O} 1 s$ for the asdeposited reference tantala sample, s12, and s22. Solid lines are collected spectra, and dashed lines are composite spectra with decomposed peaks.
TABLE IV. Peak positions from fitting results for Ta $4 f$ and O $1 s$ peaks.

\begin{tabular}{lcccc}
\hline \hline & Ta $4 f$ & Ta $4 f$ & & $\mathrm{O}_{\text {surface }}$ \\
Sample & $5 / 2(\mathrm{eV})$ & $7 / 2(\mathrm{eV})$ & $\mathrm{O} 1 \mathrm{~s}(\mathrm{eV})$ & $1 s(\mathrm{eV})$ \\
\hline Reference & $27.9 \pm 0.02$ & $26.0 \pm 0.03$ & $530.4 \pm 0.04$ & $531.9 \pm 0.06$ \\
s12 & $27.9 \pm 0.03$ & $26.0 \pm 0.01$ & $530.4 \pm 0.03$ & $531.9 \pm 0.02$ \\
s22 & $27.9 \pm 0.02$ & $26.0 \pm 0.02$ & $530.4 \pm 0.01$ & $531.9 \pm 0.05$ \\
\hline \hline
\end{tabular}

Veeco Spector ${ }^{\circledR}$ with planetary rotation at a deposition rate of $2 \AA / \mathrm{s}$ and with an absorption loss of $3 \mathrm{ppm}$ at $1064 \mathrm{~nm}$. The Ta $4 f$ doublet peaks were at 27.9 and $26.0 \mathrm{eV}$ for this reference sample (Table IV), and the values are consistent with binding energies of $\mathrm{Ta}$ in $\mathrm{Ta}_{2} \mathrm{O}_{5}[27,28]$. No suboxide peak was identified. The $\mathrm{O} 1 \mathrm{~s}$ peak was decomposed into one sharp peak at $530.4 \mathrm{eV}$ and one broad peak at $531.9 \mathrm{eV}$. The latter was attributed to surface contamination [27]. The XPS spectra of s12 and s22, which as will be shown in Sec. III. C have the largest and smallest mechanical loss, are displayed in Fig. 2. For both Ta $4 f$ doublets and $\mathrm{O} 1 \mathrm{~s}$ peaks, the peak center did not shift from that of the reference sample (Table IV). These results indicate that, within the sensitivity of XPS, there are no significant changes in bonding states in tantala samples prepared with and without ion-assisted bombardment.

\section{Mechanical loss properties}

The set of IBS conditions that were selected to assess modifications to mechanical loss include i) variations in the projectile mass, i.e., the mass of the assist ions; ii) variations in the assist ion energy; and iii) variations in the ratio between the number of sputtered particles and the number of assist ions arriving at the substrate surface. The implications these parameters have on surface diffusion are discussed in the next section.

The loss angles of the two control samples c1 and c2 deposited with no assist ion bombardment are similar, $8.5 \times 10^{-4}$ and $8.4 \times 10^{-4}$ rad for $\mathrm{c} 1$ and $\mathrm{c} 2$, respectively. These values are represented in Figs. $3-6$ by green dotted lines.

The coatings mechanical loss vs ion mass is plotted in Fig. 3. Samples s13 and s24 were deposited with $100 \mathrm{eV}$ assist ions and similar particle-to-ion arrival ratios, except that the assist ions were $\mathrm{Ar}^{+}$for $\mathrm{s} 13$ and $\mathrm{Xe}^{+}$for $\mathrm{s} 24$. The coatings loss angle shows no trend with ion mass within one standard deviation.

In Fig. 4, we compare the loss angles of samples s23 and s24 that were bombarded with $\mathrm{Xe}^{+}$of 50 and $100 \mathrm{eV}$ energy and similar particle-to-ion arrival ratios. With higher energy, the $\mathrm{Xe}^{+}$were more likely to increase the surface diffusivity of the adsorbed particles and affect the coatings loss angle. Nevertheless, this trend was not observed.

The loss angle of samples bombarded with $\mathrm{Ar}^{+}$with different particle-to-ion arrival ratios is shown in Fig. 5. 


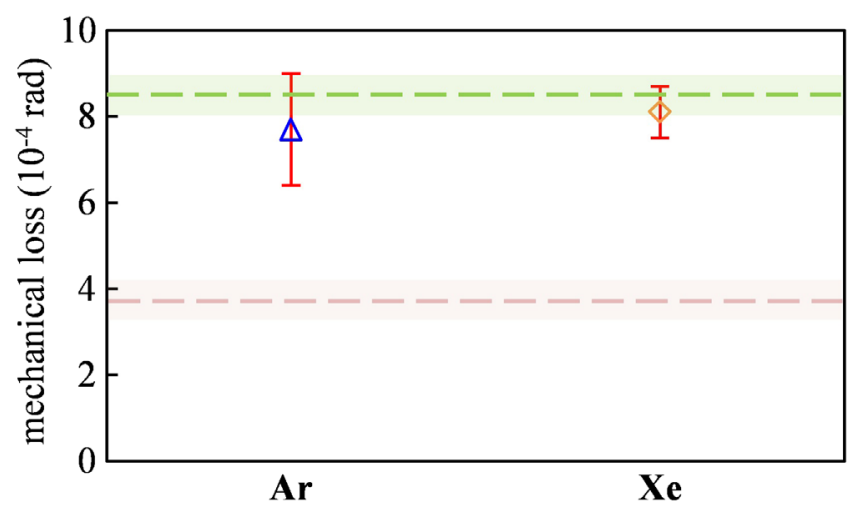

FIG. 3. Loss angles of samples bombarded by Ar and Xe ions. The ion energy was $100 \mathrm{eV}$ for both samples. The particle-to-ion arrival ratios are almost identical. s13 was bombarded with $\mathrm{Ar}^{+}$, while s24 was bombared with $\mathrm{Xe}^{+}$. The green and red dotted lines represent the loss angle level of the as-deposited control samples and the samples after annealing, respectively. The shading indicates the experimental error.

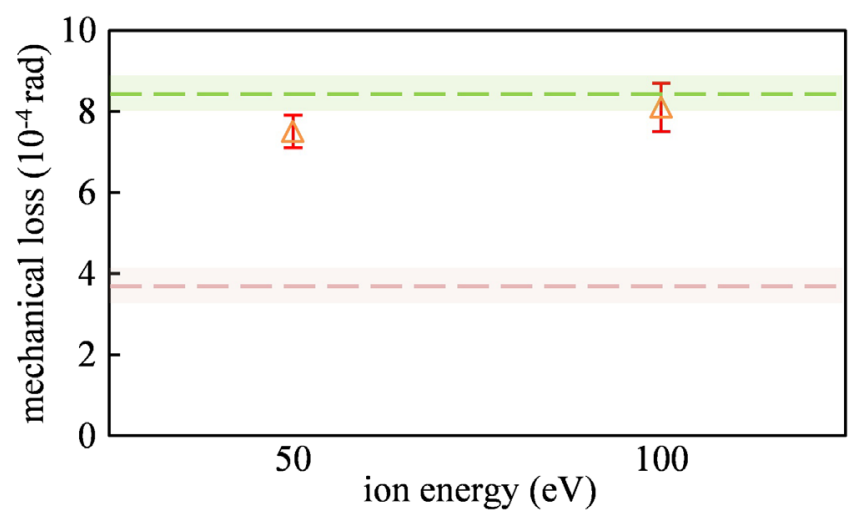

FIG. 4. Loss angles of tantala coatings bombarded by $\mathrm{Xe}^{+}$ions of different energy. The particle-to-ion arrival ratio was kept constant. The assist ion energy was $50 \mathrm{eV}$ for s 23 and $100 \mathrm{eV}$ for s24. The green and red dotted lines represent the loss angle level of the as-deposited control samples and the samples after annealing, respectively. The shading indicates the experimental error.

For s11, s12, and s13, the particle-to-ion arrival ratio decreases from $22 / 1$ to $5 / 1$. Comparison of the coatings loss angles shows no systematic trend with particle-to-ion arrival ratio. A similar behavior of the coatings loss angle is shown in Fig. 6 for $\mathrm{Xe}^{+}$assist bombardment with a particle-to-ion arrival ratio varying from $4 / 1$ to $40 / 1$.

Following these measurements, all tantala thin film samples were annealed. Regardless of the initial loss angle values, for all $\mathrm{Ar}^{+}$and $\mathrm{Xe}^{+}$bombardment conditions, the annealed tantala samples reached their lowest loss angle of approximately $4 \times 10^{-4} \mathrm{rad}$, represented by the red dotted line in Figs. 3-6, which is in the typical range for annealed tantala films deposited by ion beam sputtering [17]. The decreased loss angle is suggested to correlate with an

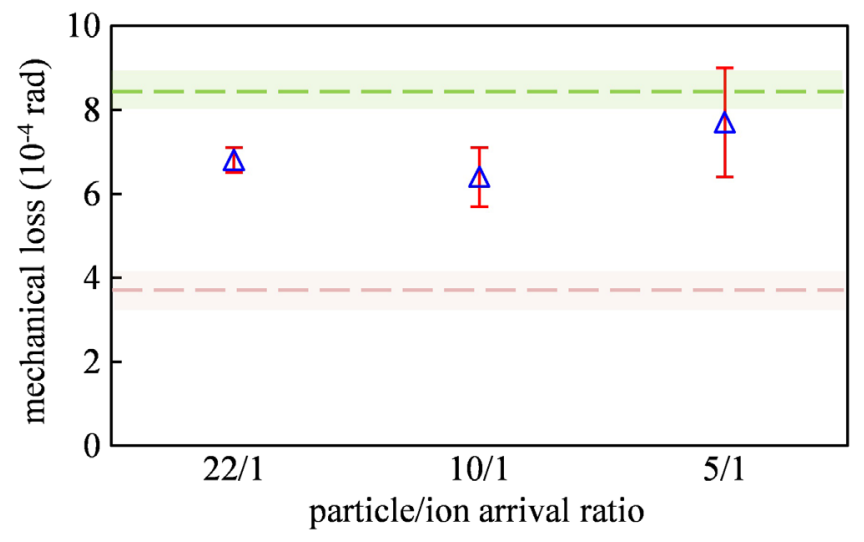

FIG. 5. Loss angle of tantala coatings bombarded with $100 \mathrm{eV}$ $\mathrm{Ar}^{+}$and different particle-to-ion arrival ratios. The green and red dotted lines represent the loss angle level of the as-deposited control samples and the samples after annealing, respectively. The shading indicates the experimental error.

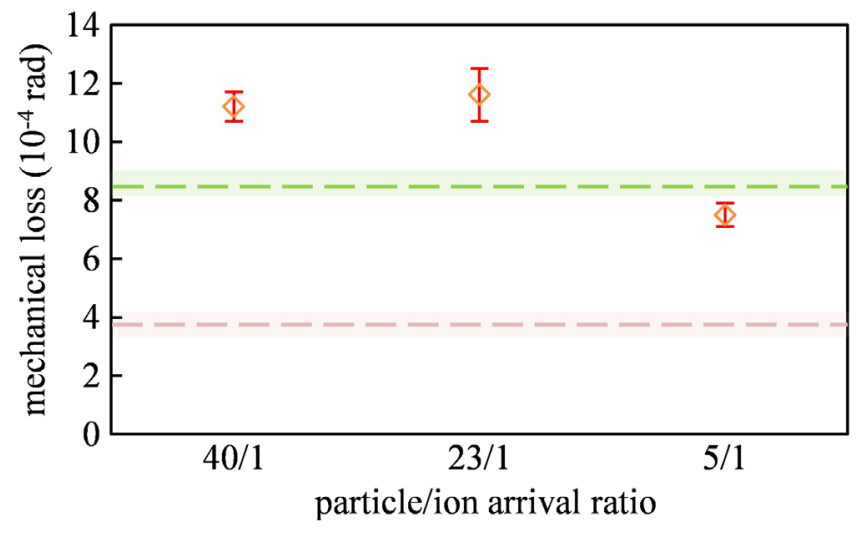

FIG. 6. Loss angles of tantala coatings bombarded with $50 \mathrm{eV}$ $\mathrm{Xe}^{+}$and different particle-to-ion arrival ratios. The green and red dotted lines represent the loss angle level of the as-deposited control samples and the samples after annealing, respectively. The shading indicates the experimental error.

increase in the medium range order (5-40 $\AA$ ) during annealing [29,30].

\section{Discussion}

Previous work on diffusion behavior of chemi-sorbed Ge on a $\mathrm{Si}$ surface at high temperatures showed that the diffusivity $D$ typically exhibits an Arrhenius behavior under ion bombardment $[18,19]$, obeying

$$
D=\theta D_{0} \exp \left(-E_{\alpha} / k T\right)
$$

where $\theta$ is the fractional coverage of mobile adatoms, $D_{0}$ is a prefactor for intrinsic surface diffusion, $E_{a}$ is the activation energy for intrinsic surface diffusion, and $T$ is the temperature. $D_{0}$ is a parameter that is empirically correlated to bulk properties, i.e., melting point, cohesive 
energy, and elastic constants [31]. It is also a function of geometrical parameters, i.e., effective jump distance, and jump-attempt frequency [32]. For surface diffusion under ion bombardment, it has been shown that $D_{0}$ is affected by ion energy, ion mass, and ion flux which is equivalent to the inverse of the particle-to-ion arrival ratio.

The results of the loss angle of tantala presented in Sec. III. C do not vary with ion mass, ion energy, or particle-to-ion arrival ratio, suggesting that surface diffusivity either does not suppress internal friction in IBS tantala or may be hindered by other mechanisms during sputtering at the conditions of the experiments.

In the IBS reactive deposition of tantala, combinations of clusters and atoms with an average energy of several $\mathrm{eVs}$ are sputtered from the target and deposit onto the substrate surface. The arriving particles adsorb and diffuse on the surface before bonding with other species. For $\mathrm{O}$ atoms with an activation energy for mass transfer diffusion of approximately $1 \mathrm{eV}$ [33] and a high fractional coverage $\theta$, the diffusivity $D$ would be strongly affected by assist ion bombardment. However, when chemi-sorbed particles are mostly clusters, $\theta$ is small. In the best case of the experiments in which the particle-to-ion arrival ratio is $4 / 1$ and assuming no more than one adatom is created by breaking $\mathrm{Ta}_{2} \mathrm{O}_{2}$ clusters, the maximum ion-induced $\theta$ is 0.06 . This, coupled with the large activation energy of approximately $2.5 \mathrm{eV}$ for clusters [34], results in insignificant cluster diffusivity, even when considering that the steady-state substrate temperature, $T$, increases to $130{ }^{\circ} \mathrm{C}$ during ion bombardment. Theoretically, an assist ion dose ten times larger than that accessible in the present experiments would be needed to increase the number of surface mobile atoms to a level that may affect variations in the coating mechanical loss, if any.

Reduced mobility of the chemisorbed particles is compounded by the limited time there is before the next layer is deposited. An $\mathrm{O}$ adatom has only $2 \mathrm{~s}$ to diffuse when the deposition rate is that of the experiments, $0.9 \AA / \mathrm{s}$. With an estimated diffusivity $D$ around $10^{-2} \mathrm{~nm}^{2} \mathrm{~s}^{-1}$ [32], the diffusion length is less than $2 \AA$ at a steady-state temperature of $130^{\circ} \mathrm{C}$. Even if one considers that at the location where a $100 \mathrm{eV}$ assist ion impinges a temperature spike that reaches $900^{\circ} \mathrm{C}$ and lasts $10^{-11} \mathrm{~s}$ is produced, the diffusion length of $\mathrm{O}$ within such short length scales is less than $0.1 \AA$. Based on this analysis, we conclude that the surface diffusivity in IBS tantala films induced by assist ion bombardment under the conditions of the experiments is not significant to affect the coatings loss angle. The lack of surface diffusivity can also explain the relatively small variation in coatings loss angle on high temperature sputtered tantala [17] and its behavior with annealing, which consistently lowered the loss angle to approximately $4 \times 10^{-4} \mathrm{rad}$ independently of deposition conditions. During a $10 \mathrm{~h}$ anneal at $500^{\circ} \mathrm{C}$, the diffusion length of an oxygen atom in the tantala film is around $700 \AA$, much larger than the few angstroms oxygen can diffuse at the conditions of our experiment.

The diffusion analysis can explain the variation of loss angle with deposition and annealing temperature of a-Si [16]. a-Si thin films deposited at room temperature and post annealed at $400^{\circ} \mathrm{C}$ have a loss angle of $0.5 \times 10^{-4} \mathrm{rad}$. The exact same value was obtained in a-Si thin films deposited at a temperature of $400{ }^{\circ} \mathrm{C}$. In this case, surface diffusion and relaxation make it possible to improve the coatings loss angle. This is in contrast with similar experiments in tantala thin films [17], which report a loss angle of $7 \times 10^{-4} \mathrm{rad}$ for a sample deposited at $400^{\circ} \mathrm{C}$ and $5 \times 10^{-4} \mathrm{rad}$ for one deposited at room temperature and annealed at $400^{\circ} \mathrm{C}$. Thus, ion bombardment could have an effect similar to high temperature annealing when atomic species are deposited at a rate that ensures surface relaxation before the atoms are buried by the next layer.

Nevertheless, one should note that surface diffusion is not the only factor that plays a role in affecting the room temperature coating mechanical loss. If a reduction in mechanical loss were associated with the restructuring of polyhedra from face and edge sharing to corner sharing during the annealing process, as recently shown in amorphous $\mathrm{Zr}$ doped $\mathrm{Ta}_{2} \mathrm{O}_{5}$ thin films [35], a significant diffusion length would not be required. The mechanism on how oxygen diffusion would facilitate the reorganization is still unclear. It should also be pointed out that $\mathrm{Ta}_{2} \mathrm{O}_{5}$ is not an ambient-pressure glass-forming oxide [36] as oxygen coordinates to an average of more than two cations. This, along with the predominant $\mathrm{Ta}-\mathrm{O}$ ionic bond character [37], forms a structure that is more difficult to modify.

The ion-induced surface diffusivity analysis with lowenergy $\mathrm{Ar}^{+}$and $\mathrm{Xe}^{+}$ions ignores possible surface and bulk modifications due to other mechanisms, such as surface relaxation via viscous flow in the bulk due to displacements produced in the collision cascade process [38]. Along with these effects, there is still mass transport due to the random removal of atoms from the flat surface and the slope- and curvature-dependent sputtering [39]. Experiments with $\mathrm{Xe}^{+}$bombardment at a high angle of incidence could allow for more energy deposited at the surface, improving adatom mobility and isolating effects from the bulk.

\section{CONCLUSIONS}

To summarize, we investigated the influence of lowenergy assist ion bombardment on the mechanical loss of optical quality tantala thin films deposited by reactive IBS. $\mathrm{Ar}^{+}$and $\mathrm{Xe}^{+}$ion beams were generated at different conditions to identify variations in the coating loss angle due to the mass and energy of the assist ion and ion dose. Most films are of excellent optical quality with an absorption loss at $1064 \mathrm{~nm}$ lower than $18 \mathrm{ppm}$, while no preferential sputtering of oxygen by the assist ions was identified. At a microscopic level, the atomic structure of the films was found to be amorphous, and the bonding states of 
Ta remained the same for samples prepared with and without assist ion bombardment. Surface diffusion and its impact on the tantala coatings loss angle were evaluated assuming an Arrhenius behavior. By comparing samples bombarded with $\mathrm{Ar}^{+}$and $\mathrm{Xe}^{+}$, no statistically significant trend in loss angle was observed with ion mass. In the bombardment with $\mathrm{Ar}^{+}$ or $\mathrm{Xe}^{+}$assist ions, the variance in the particle-to-ion arrival ratio did not result in a significant difference in the coating loss angle, either. The influence of assist ion bombardment on coatings loss angle is suggested to be minor.

The lack of evidence in ion-induced diffusivity affecting mechanical loss could be explained by the fact that chemisorbed particles are mainly clusters that are essentially immobile with a small proportion of chemi-sorbed adatoms. The adatoms have also insufficient time to rearrange before being buried by the next layer within the timescales of the deposition process in these experiments.

\section{ACKNOWLEDGMENTS}

This work is supported by the National Science Foundation LIGO program through Grant No. 1710957. We also acknowledge the support of the LIGO Scientific Collaboration (LSC) Center for Coatings Research, jointly funded by the National Science Foundation (NSF) and the Gordon and Betty Moore Foundation.
[1] B. P. Abbott et al. (LIGO Scientific and Virgo Collaborations), Phys. Rev. Lett. 116, 131103 (2016).

[2] B. P. Abbott et al. (LIGO Scientific and Virgo Collaborations), Phys. Rev. Lett. 116, 061102 (2016).

[3] B. P. Abbott et al. (LIGO Scientific and Virgo Collaborations), Phys. Rev. Lett. 119, 161101 (2017).

[4] G. M. Harry, A. M. Gretarsson, P. R. Saulson, S. E. Kittelberger, S. D. Penn, W. J. Startin, S. Rowan, M. M. Fejer, D. Crooks, G. Cagnoli et al., Classical Quantum Gravity 19, 897 (2002).

[5] J. Miller, L. Barsotti, S. Vitale, P. Fritschel, M. Evans, and D. Sigg, Phys. Rev. D 91, 062005 (2015).

[6] G. M. Harry, M. R. Abernathy, A. E. Becerra-Toledo, H. Armandula, E. Black, K. Dooley, M. Eichenfield, C. Nwabugwu, A. Villar, D. Crooks et al., Classical Quantum Gravity 24, 405 (2007).

[7] M. Granata, E. Saracco, N. Morgado, A. Cajgfinger, G. Cagnoli, J. Degallaix, V. Dolique, D. Forest, J. Franc, C. Michel et al., Phys. Rev. D 93, 012007 (2016).

[8] L. Pinard, C. Michel, B. Sassolas, L. Balzarini, J. Degallaix, V. Dolique, R. Flaminio, D. Forest, M. Granata, B. Lagrange et al., Appl. Opt. 56, C11 (2017).

[9] Y. T. Liu and K. S. Thorne, Phys. Rev. D 62, 122002 (2000).

[10] Y. Levin, Phys. Lett. A 372, 1941 (2008).

[11] A. Amato, G. Cagnoli, M. Canepa, E. Coillet, J. Degallaix, V. Dolique, D. Forest, M. Granata, V. Martinez, C. Michel et al., J. Phys. Conf. Ser. 957, 012006 (2018).

[12] R. Flaminio, J. Franc, C. Michel, N. Morgado, L. Pinard, and B. Sassolas, Classical Quantum Gravity 27, 084030 (2010).

[13] R. Bassiri, K. Evans, K. Borisenko, M. Fejer, J. Hough, I. MacLaren, I. Martin, R. Route, and S. Rowan, Acta Mater. 61, 1070 (2013).

[14] T. Pérez-Castañeda, C. Rodríguez-Tinoco, J. RodríguezViejo, and M. A. Ramos, Proc. Natl. Acad. Sci. U.S.A. 111, 11275 (2014).

[15] X. Liu, D. R. Queen, T. H. Metcalf, J.E. Karel, and F. Hellman, Phys. Rev. Lett. 113, 025503 (2014).
[16] R. Birney, J. Steinlechner, Z. Tornasi, S. MacFoy, D. Vine, A. Bell, D. Gibson, J. Hough, S. Rowan, P. Sortais et al., Phys. Rev. Lett. 121, 191101 (2018).

[17] G. Vajente, R. Birney, A. Ananyeva, S. Angelova, R. Asselin, B. Baloukas, R. Bassiri, G. Billingsley, M. Fejer, D. Gibson et al., Classical Quantum Gravity 35, 075001 (2018).

[18] R. Ditchfield and E. G. Seebauer, Phys. Rev. Lett. 82, 1185 (1999).

[19] R. Ditchfield and E. G. Seebauer, Phys. Rev. B 63, 125317 (2001).

[20] E. Hirsch and I. Varga, Thin Solid Films 69, 99 (1980).

[21] P. Langston, E. Krous, D. Schiltz, D. Patel, L. Emmert, A. Markosyan, B. Reagan, K. Wernsing, Y. Xu, Z. Sun et al., Appl. Opt. 53, A276 (2014).

[22] R. Bassiri, K. Borisenko, D. Cockayne, J. Hough, I. MacLaren, and S. Rowan, Appl. Phys. Lett. 98, 031904 (2011).

[23] A. Alexandrovski, M. Fejer, A. Markosian, and R. Route, in Solid State Lasers XVIII: Technology and Devices (International Society for Optics and Photonics, San Jose, CA, 2009), Vol. 7193, p. 71930D.

[24] G. Vajente, A. Ananyeva, G. Billingsley, E. Gustafson, A. Heptonstall, E. Sanchez, and C. Torrie, Rev. Sci. Instrum. 88, 073901 (2017).

[25] LIGO Scientific Collaboration, Classical Quantum Gravity 32, 074001 (2015).

[26] I. Perez, J. L. E. Carrejo, V. Sosa, F. G. Perera, J. R. F. Mancillas, J. T. E. Galindo, and C. I. R. Rodríguez, J. Alloys Compounds 712, 303 (2017).

[27] E. Atanassova, G. Tyuliev, A. Paskaleva, D. Spassov, and K. Kostov, Appl. Surf. Sci. 225, 86 (2004).

[28] R. Simpson, R. G. White, J. F. Watts, and M. A. Baker, Appl. Surf. Sci. 405, 79 (2017).

[29] R. Bassiri, F. Liou, M. R. Abernathy, A. C. Lin, N. Kim, A. Mehta, B. Shyam, R. L. Byer, E. K. Gustafson, M. Hart et al., APL Mater. 3, 036103 (2015). 
[30] M. J. Hart, R. Bassiri, K. B. Borisenko, M. Véron, E. F. Rauch, I. W. Martin, S. Rowan, M. M. Fejer, and I. MacLaren, J. Non-Cryst. Solids 438, 10 (2016).

[31] D. Gupta, Diffusion Processes in Advanced Technological Materials (Springer Science \& Business Media, New York, 2010).

[32] R. Nakamura, T. Toda, S. Tsukui, M. Tane, M. Ishimaru, T. Suzuki, and H. Nakajima, J. Appl. Phys. 116, 033504 (2014).

[33] Y. Du, Z. Dohnálek, and I. Lyubinetsky, J. Phys. Chem. C 112, 2649 (2008).

[34] E. G. Seebauer and C. Allen, Prog. Surf. Sci. 49, 265 (1995).
[35] K. Prasai, J. Jiang, A. Mishkin, B. Shyam, S. Angelova, R. Birney, D. Drabold, M. Fazio, E. Gustafson, G. Harry et al., Phys. Rev. Lett. 123, 045501 (2019).

[36] O. L. Alderman, C. Benmore, J. Neuefeind, E. Coillet, A. Mermet, V. Martinez, A. Tamalonis, and R. Weber, Phys. Rev. Mater. 2, 043602 (2018).

[37] N. Kim and J. F. Stebbins, Chem. Mater. 23, 3460 (2011).

[38] T. Mayer, E. Chason, and A. Howard, J. Appl. Phys. 76, 1633 (1994).

[39] R. M. Bradley and J. M. Harper, J. Vac. Sci. Technol. A 6, 2390 (1988). 